\title{
Accounting for Future Redesign in the Optimization of an Integrated Thermal Protection System
}

\author{
Diane Villanueva ${ }^{1,2 *}$ Raphael T. Haftka ${ }^{1 \dagger}$, and Bhavani V. Sankar ${ }^{1 \ddagger}$ \\ ${ }^{1}$ University of Florida, Gainesville, FL, 32611, USA \\ ${ }^{2}$ École Nationale Superiéure des Mines de Saint-Étienne, Saint-Etienne, France
}

\begin{abstract}
Most components undergo tests after they are designed and are redesigned if necessary. Tests help designers find unsafe and overly conservative designs, and redesign can restore safety or reduce mass. In general, the changes to the mass and reliability of the design after the test and redesign are not considered. This paper examines the effect of controlling design parameters and the parameters that control the test and redesign when optimizing the design on an integrated thermal protection system of a space vehicle. This study explores the tradeoff between the probability of redesign and the mass at the design stage. We observed that to minimize mass, designs should initially satisfy safety requirements such that most redesigns concern overly conservative designs. The additional knowledge gained from the test allows the designer to consider a smaller safety margin for the redesign to reduce the mass.
\end{abstract}

\section{Nomenclature}

$\begin{array}{ll}d & =\text { design variable } \\ e_{c} & =\text { computational error } \\ e_{x} & =\text { experimental error } \\ f(T) & =\text { probability distribution of the temperature } \\ m & =\text { mass per unit area, } \frac{\mathrm{kg}}{\mathrm{m}^{2}} \\ p_{f} & =\text { probability of failure, } \% \\ r & =\text { random variable } \\ S & =\text { safety margin } \\ \Delta T & =\text { change in temperature, } \mathrm{K} \\ T & =\text { temperature, } \mathrm{K} \\ \text { subscripts } & \\ 0 & =\text { initial } \\ \text { calc } & =\text { calculated } \\ \text { corr } & =\text { corrected } \\ \text { nom } & =\text { nominal } \\ \text { meas } & =\text { measured } \\ \text { test } & =\text { test article } \\ \text { true } & =\text { true }\end{array}$

\footnotetext{
* Graduate Research Assistant, Mechanical and Aerospace Engineering/Institut Henri Fayol, AIAA student member

${ }^{\dagger}$ Distinguished Professor, Mechanical and Aerospace Engineering, AIAA Fellow

‡Ebaugh Professor, Mechanical and Aerospace Engineering, AIAA Associate Fellow
} 


\section{Introduction}

In reliability based design optimization, uncertainties are considered when calculating the reliability of the structure. In the design process, uncertainty is often compensated for with safety factors and knockdown factors. However, after design, it is customary for the component to undergo various uncertainty reduction measures (URMs). Examples of URMs in the aerospace field include thermal and structural testing, inspection, health monitoring, maintenance, and improved analysis and failure modeling. Since most components undergo these URMs, it would be beneficial to include their effects in the design process.

In recent years, there has been a movement to quantify the effect of URMs on the safety of the product over its life cycle. Much work has been completed in the areas of inspection and maintenance for structures under fatigue loading. ${ }^{1-4}$ Studies by Acar et al. ${ }^{5}$ investigated the effects of future tests and redesign on the final distribution of failure stress and structural design with varying numbers of tests at the coupon, element, and certification levels. Sankararaman et al. ${ }^{6}$ proposed an optimization algorithm of test resource allocation for multi-level and coupled systems.

Previously, Villanueva et al. ${ }^{7}$ proposed a method to incorporate the effect of a single future thermal test followed by redesign on the initial reliability estimates of an integrated thermal protection system (ITPS). An ITPS is a structure on a reusable launch vehicle that simultaneously provides protection from aerodynamic heating during reentry, while working as a load bearing structure. Monte Carlo sampling of the assumed computational and experimental errors was used to sample future test alternatives, or the possible outcomes of the future test. Using the future alternatives, the methodology included two methods of calibration and redesign, needed when the test result indicates that the design is unacceptable. It was observed that the deterministic approach to calibration and redesign, which acts to restore the original (designed) safety margin, leads to a greatly reduced probability of failure after the test and redesign, a reduction that usually is not quantified. A probabilistic approach was also presented, which provided a way to more accurately estimate the probability of failure after the test and allowed the tradeoff of weight against performing additional tests. Matsumura et al. ${ }^{8}$ extended the methodology to include additional failure modes of the ITPS.

This paper examines the effect of controlling design parameters and the parameters that control the future test and redesign when optimizing the design of the ITPS. The focus is on tuning the redesign conditions (i.e., the rules that dictate whether or not redesign is performed) along with the design such that both the mass and probability of an unacceptable test result are minimized. The desire to keep probability of redesign small is obvious in that redesign can lead to project delays and increase costs. On the other hand, a reduction in mass is attractive, especially for space vehicles which have extremely large per-weight launch costs. This study will explore the tradeoff between the probability of redesign and the mass at the design stage.

\section{A. Integrated Thermal Protection Shield Description}

Figure 1 shows the ITPS panel that is studied, which is a corrugated core sandwich panel concept. The design consists of a top face sheet and webs made of titanium alloy (Ti-6Al-4V), and a bottom face sheet made of beryllium. Saffil ${ }^{\circledR}$ foam is used as insulation between the webs. The relevant geometric variables of the ITPS design are also shown on the unit cell in Figure 1. These variables are the top face thickness $\left(t_{T}\right)$, bottom face thickness $\left(t_{B}\right)$, thickness of the foam $\left(d_{S}\right)$, web thickness $\left(t_{w}\right)$, corrugation angle $(\theta)$, and length of unit cell $(2 p)$. The mass per unit area is calculated using Eq.(1)
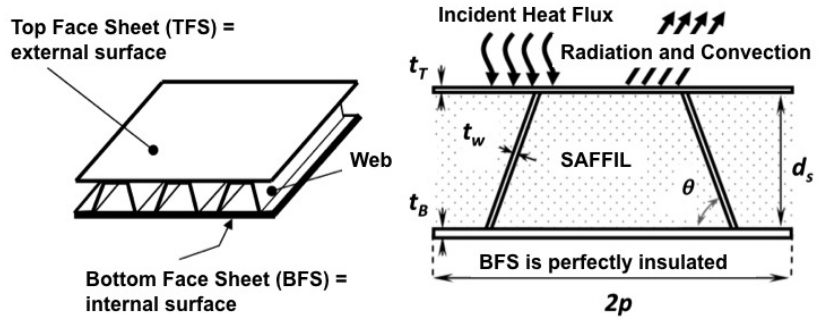

Figure 1. Corrugated core sandwich panel ITPS concept

$$
m=\rho_{T} t_{T}+\rho_{B} t_{B}+\frac{\rho_{w} t_{w} d_{S}}{p \sin \theta}
$$

where $\rho_{T}, \rho_{B}$, and $\rho_{w}$ are the densities of the materials that make up the top face sheet, bottom face sheet, and web, respectively. Additional information on the integrated thermal protection system is provided in the Appendix. 
In this study, we consider thermal failure to occur when the temperature of the bottom face sheet exceeds an allowable temperature. We assume that tests of the structure will be conducted to verify the design. Observed data from the test will be utilized to calibrate errors in analytical calculations.

\section{Analysis and Post-Design Test with Redesign}

It is assumed that an analyst has a computational model by which to calculate the change in the temperature of the bottom face sheet of the ITPS, $\Delta T_{\text {calc }}$, for a design described by design variables $d$ and random variables $r$. The randomness is due to variabilities in material properties, manufacturing, and environmental effects. Using $\Delta T_{\text {calc }}$, the calculated temperature is defined as

$$
T_{\text {calc }}\left(d, r, v_{0}\right)=T_{0}\left(1-v_{0}\right)+\Delta T_{\text {calc }}(d, r)
$$

where $T_{0}$ is the initial temperature of the bottom face sheet, which also has variability represented by $v_{0}$.

The design is obtained via a deterministic optimization problem which requires that the calculated temperature be less than or equal to some deterministic allowable temperature $T_{\text {allow }}^{\text {det }}$ by some a safety margin $S_{1}$ as shown in Eq.(3).

$$
\begin{array}{ll}
\min _{d=\left\{t_{w}, t_{B}, d_{S}\right\}} & m(d) \\
\text { subject to } & T_{0}+\Delta T_{\text {calc }}\left(d, r_{\text {nom }}\right)+S_{1} \leq T_{\text {allow }}^{\text {det }} \\
& t_{w, l} \leq t_{w} \leq t_{w, u} \\
& t_{B, l} \leq t_{B} \leq t_{B, u} \\
& d_{S, l} \leq d_{S} \leq d_{S, u}
\end{array}
$$

Note that for the deterministic design, the random variables are held at the nominal value $r_{n o m}$ and the variability in the initial temperature is zero. The subscripts $l$ and $u$ on the design variables represent the lower and upper bounds, respectively.

After the design stage, a test is conducted to verify the chosen design. The test is performed on a test article described by $d_{\text {test }}$ and $r_{\text {test }}{ }^{\mathrm{a}}$ and an experimentally measured change in temperature, $\Delta T_{\text {meas }}$, is found. For this test design, $\Delta T_{\text {calc }}\left(d_{\text {test }}, r_{\text {test }}\right)$ and $T_{\text {calc }}\left(d_{\text {test }}, r_{\text {test }}\right)$ are also calculated.

As a means of calibration, the experimentally measured and calculated temperatures can be used in the form of a correction factor $\theta$ for the computational model. That is, the corrected calculated temperature is given as

$$
\begin{aligned}
T_{\text {calc,corr }}\left(d, r, v_{0}\right) & =T_{0}\left(1-v_{0}\right)+\theta \Delta T_{\text {calc }}(d, r) \\
\text { where } \theta & =\frac{\Delta T_{\text {meas }}}{\Delta T_{\text {calc }\left(d_{\text {test }}, r_{\text {test }}\right)}}
\end{aligned}
$$

Note that this results in an updated distribution of the corrected-calculated temperature.

Should the test result show that a design is unacceptable, redesign occurs. The criterion for redesign is based on the safety margin of the corrected calculated temperature of the original design. The lower and upper limits of the safety margin of the corrected temperature are represented with $S_{2}$ and $S_{3}$, respectively. This is expressed as

$$
\begin{aligned}
\text { Redesign if: } & T_{\text {allow }}^{\text {det }}-\left(T_{0}+\theta \Delta T_{\text {calc }}\left(d, r_{\text {nom }}\right)\right) \leq S_{2} \\
& \text { or } T_{\text {allow }}^{\text {det }}-\left(T_{0}+\theta \Delta T_{\text {calc }}\left(d, r_{\text {nom }}\right)\right) \geq S_{3}
\end{aligned}
$$

Deterministic redesign is performed so that the corrected calculated temperature of the redesign (with the correction factor) is less than or equal to the allowable temperature by a safety margin $S_{4}$. This safety margin $S_{4}$ does not necessarily need to be equal to the initial safety margin $S_{1}$. For example, since more information is gained from the test, the designer may choose to design to save weight by reducing the safety margin. This can formulated into an optimization problem to minimize the mass given a constraint on the

\footnotetext{
${ }^{a}$ It is assumed that the test article design is accurately measured such that $r_{t e s t}$ is deterministic and there is no variability in the initial temperature.
} 
corrected calculated temperature of the new redesign, where the design variables are the geometry.

$$
\begin{array}{ll}
\min _{d=\left\{t_{w}, t_{B}, d_{S}\right\}} & m(d) \\
\text { subject to } & T_{0}+\theta \Delta T_{\text {calc }}\left(d, r_{\text {nom }}\right)_{\text {redesign }}+S_{4} \leq T_{\text {allow }}^{\text {det }} \\
& t_{w, l} \leq t_{w} \leq t_{w, u} \\
& t_{B, l} \leq t_{B} \leq t_{B, u} \\
& d_{S, l} \leq d_{S} \leq d_{S, u}
\end{array}
$$

\section{Uncertainty Definition}

Oberkampf et al. ${ }^{9}$ provided an analysis of different sources of uncertainty in engineering modeling and simulation, which was simplified by Acar et al. ${ }^{10}$ We use classification similar to Acar's to categorize types of uncertainty as errors (uncertainties that apply equally to every ITPS) or variability (uncertainties that vary in each individual ITPS). We further describe errors as epistemic and variability as aleatory.

Variability is modeled as random uncertainties that can be modeled probabilistically. We simulate the variability through a Monte Carlo simulation (MCS) that generates values of the random variables $r$ based on an estimated distribution and calculates the change bottom face sheet temperature $\Delta T_{\text {calc }}$. In addition, we sample the variability in the initial temperature $v_{0}$. This forms the temperature $T_{\text {calc }}$ for each sample, generating the probability distribution function. The calculated temperature distribution that reflects the random variability is denoted $f_{\text {calc }}(T)$. Additionally, we have variability in the allowable temperature $T_{\text {allow }}$.

In contrast to variability, errors are fixed for a given ITPS and the true values are largely unknown, so they can be modeled probabilistically as well. We have classified two sources of error, which are described in Table 1.

Table 1. Description of Errors

\begin{tabular}{ll}
\hline Symbol & Description \\
\hline$e_{c}$ & computational error due to modeling of the temperature change $\Delta T_{\text {calc }}$ \\
$e_{x}$ & experimental error in measuring $\Delta T_{\text {meas }}$ \\
\hline
\end{tabular}

In estimating the temperature of a design, the error must also be considered. As previously described, the calculated temperature distribution $f_{\text {calc }}(T)$ of the design reflects random variability. If the true value of the computational error is known, then the true temperature distribution, $f_{\text {true }}(T)$, associated with $f_{\text {calc }}(T)$ is known, as shown in Fig. 2(a). The true temperature still has randomness due to the variabilities.

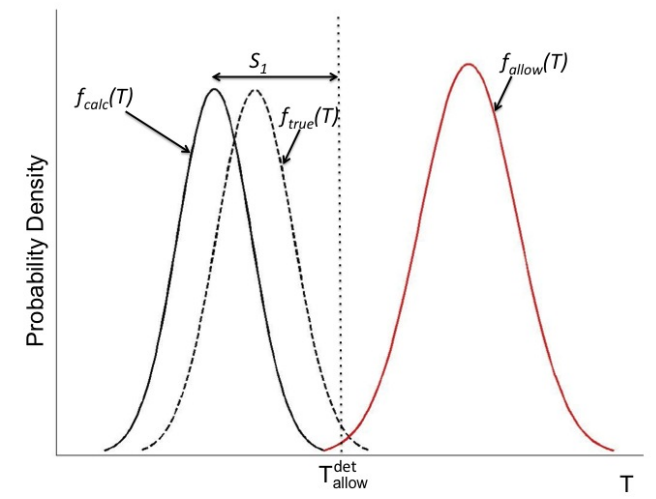

(a) Calculated, true, and allowable temperature distri- $($ b) butions

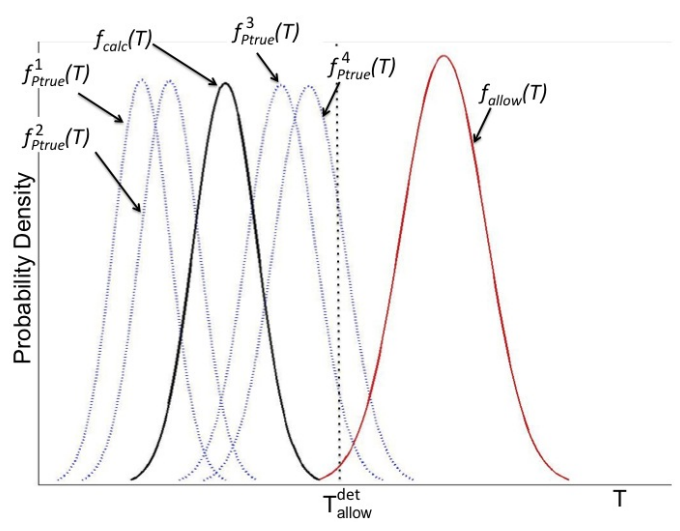

(b) Calculated, allowable, and sampled possible true temperature distributions

Figure 2. Example illustrating (a) known calculated and allowable temperature distributions and unknown true distribution, (b) sampling to obtain possible true temperature distributions 
Since the error is unknown and modeled probabilistically, we instead sample the computational error to create several possible distributions of the true temperature distributions, $f_{\text {Ptrue }}^{i}(T)$ corresponding to the $i$ th sample of $e_{c}$. This sampling is illustrated in Fig. 2(b).Using the allowable temperature distribution, the probability of failure can be calculated for each sample of the computational error. This forms a distribution of the probability of failure.

\section{Distribution of the Probability of Failure}

The true temperature for a design described by geometric design variables $d$ and random variables $r$ can be defined as

$$
T_{\text {true }}\left(d, r, v_{0}\right)=T_{0}\left(1-v_{0}\right)+\left(1-e_{c, \text { true }}\right) \Delta T_{\text {calc }}(d, r)
$$

The limit state for the probability of failure takes into account the variability in the allowable temperature $^{\mathrm{b}}$ along with the distribution of the true temperature. The limit state equation $g$ is formulated as the difference between a capacity $C$ and response $R$ as shown in Eq.(8).

$$
g_{\text {true }}=T_{\text {allow }}-T_{\text {true }}\left(d, r, v_{0}\right)=C-R
$$

Using the limit state equation, the probability of failure is calculated using Separable Monte Carlo. ${ }^{11}$ The probability of failure $p_{f}$ is calculated with Eq.(9), where $M$ and $N$ are the number of capacity and response samples, respectively. The indicator function $I$ is 1 if the $g$ is less than zero and 0 otherwise.

$$
p_{f}=\frac{1}{M N} \sum_{i=1}^{N} \sum_{j=1}^{M} I\left[g_{\text {true }}\left(C_{j}, R_{i}\right)<0\right]
$$

As described in the previous section, a distribution of the probability of failure can be formed by sampling the computational error for $e_{c, t r u e}$ and calculating the probability of failure for each sample.

\section{Simulating Future Processes at the Design Stage}

Monte Carlo sampling of the true values of the errors from the assumed distributions is used to simulate the future test and redesign alternatives for a candidate design. The steps to simulate a single alternative of the future test with possible redesign are listed below:

1. Sample the true errors from assumed distributions

2. Construct the virtual test article and use the true $e_{c}$ and $e_{x}$ samples to simulate a test result and correction factor $\theta$

3. Apply the correction factor based on the test result to $\Delta T_{\text {calc }}$

4. Evaluate if redesign is necessary based on $S_{2}$ and $S_{3}$, then redesign, if necessary

5. Evaluate mass and probability of failure using surrogates

To simulate another alternative, the true errors are re-sampled and the process is repeated. Therefore, for $n$ possible outcomes of a future test, we sample $n$ sets of the errors, and obtain $n$ true probabilities of failure and up to $n$ updated designs. Note that there is a single initial design, but if $k$ of the $n$ cases are re-designed we will end up with up to $k+1$ different designs.

\section{Optimization of the Geometry and the Redesign Procedure}

\section{A. Problem Description}

We explored the effect of the future test and redesign when optimizing mass with the safety margins $S_{1}, S_{2}$, $S_{3}$, and $S_{4}$ as design variables. This was done through an optimization problem that minimizes the mean

\footnotetext{
bThe absence of the superscript "det" for $T_{\text {allow }}$ denotes the allowable temperature with variability to distinguish it from the deterministic allowable temperature $T_{\text {allow }}^{\text {det }}$.
} 
mass $\mu_{m}$ and probability of redesign $p_{r e}$ subject to constraints on the mean probability of failure $\mu_{p_{f}}$, the 95th percentile of the probability of failure $P_{95}\left(p_{f}\right)$, the ranges of the safety margins, and the ranges of the design variables. The formulation is given in Eq.(10).

$$
\begin{array}{cl}
\min _{S_{1}, S_{2}, S_{3}, S_{4}} & \mu_{m}, p_{r e} \\
\text { subject to } & \mu_{p_{f}} \leq 0.1 \% \\
& P_{95}\left(p_{f}\right) \leq 0.5 \% \\
& 35 \leq S_{i} \leq 65 \text { for } i=1,4 \\
& S_{1}-35 \leq S_{2} \leq S_{1} \\
& S_{1} \leq S_{3} \leq S_{1}+35 \\
& 1.24 \mathrm{~mm} \leq t_{w} \leq 1.77 \mathrm{~mm} \\
& 4.94 \mathrm{~mm} \leq t_{B} \leq 7.06 \mathrm{~mm} \\
& 49.9 \mathrm{~mm} \leq d_{S} \leq 71.3 \mathrm{~mm}
\end{array}
$$

The constraints on $S_{1}$ and $S_{4}$ restrict the two values to be within the window of 35 to $65 \mathrm{~K}$, and they are not constrained to have equal values. The constraints on $S_{2}$ and $S_{3}$ restrict the acceptable values of the safety margin after correction to within $35 \mathrm{~K}$ of $S_{1}$.

Table 2. Bounds of computational and experimental errors

\begin{tabular}{ccc}
\hline Error & Distribution & Bounds \\
\hline$e_{c}$ & Uniform & \pm 0.12 \\
$e_{x}$ & Uniform & \pm 0.03 \\
\hline
\end{tabular}

For this problem, the computational and experimental errors were distributed as described in Table 2. Given these distributions, the correction factor $\theta$ ranged from 0.85 to 1.15 . The distributions of the variables with uncertainty due to variability are provided in the Appendix.

To reduce the computational cost of simulating a future test, surrogates of the mass and reliability index were developed. The reliability index $\beta$ is related to the probability of failure by $p_{f}=\Phi(-\beta)$, where $\Phi$ is the standard normal cumulative density function. For example, for a probability of failure of $0.1 \%$, the reliability index is 3.72 . The development of these surrogates is described in the Appendix.

The problem in Eq.(10) was solved by forming a cloud of 10,000 points using Latin Hypercube sampling of the design variables $S_{1}, S_{2}, S_{3}$, and $S_{4}$. For each set of design variables, 10,000 future alternatives were sampled to obtain the distributions of the mass and probability of failure, and the probability of redesign. The probability of redesign was calculated by dividing the number of redesigns by the total number of alternatives (i.e., 10,000). The set of points that satisfied the constraints on the probability of failure was found, and, from this set of feasible points, we formed the Pareto front for minimum probability of redesign and mean mass after redesign.

\section{B. Results}

As a point of comparison, we also found the Pareto front for minimum mass and mean probability of failure without redesign (assuming no tests are performed and no redesign). These were obtained for values of $S_{1}$ ranging from 35 to $65 \mathrm{~K}$. The Pareto front is shown in Fig. 3. The points on the Pareto front are described in the Appendix. The design with the minimum mass of $24.70 \mathrm{~kg} / \mathrm{m}^{2}$ was found with a safety margin $S_{1}$ of $48.9 \mathrm{~K}$. The mean probability of failure was $0.098 \%$ and the 95 th percentile was $0.5 \%$.

The Pareto front for minimum probability of redesign and mean mass after redesign is displayed in Fig. 4. The points on this Pareto front are also detailed in the Appendix. We observed that the majority of infeasible design have small mean mass values. This is obvious as lighter designs will have less insulation, and thus higher temperatures and larger probabilities of failure. Reductions in mean mass after redesign were observed for probabilities of redesign greater than $10 \%$. The mean mass values after redesign at these points were less than the minimum mass of $24.70 \mathrm{~kg} / \mathrm{m}^{2}$ obtained when redesign was not allowed.

Figure 5 shows the percent reduction in mass and probability of failure after redesign for the Pareto front. While the reductions in mean mass were less than $5 \%$ and the median mass remained mostly the same, we observed large reductions in the mean and 95th percentile of the probability of failure. However, the increase in median probability of failure was large, particularly at large probabilities of redesign. 


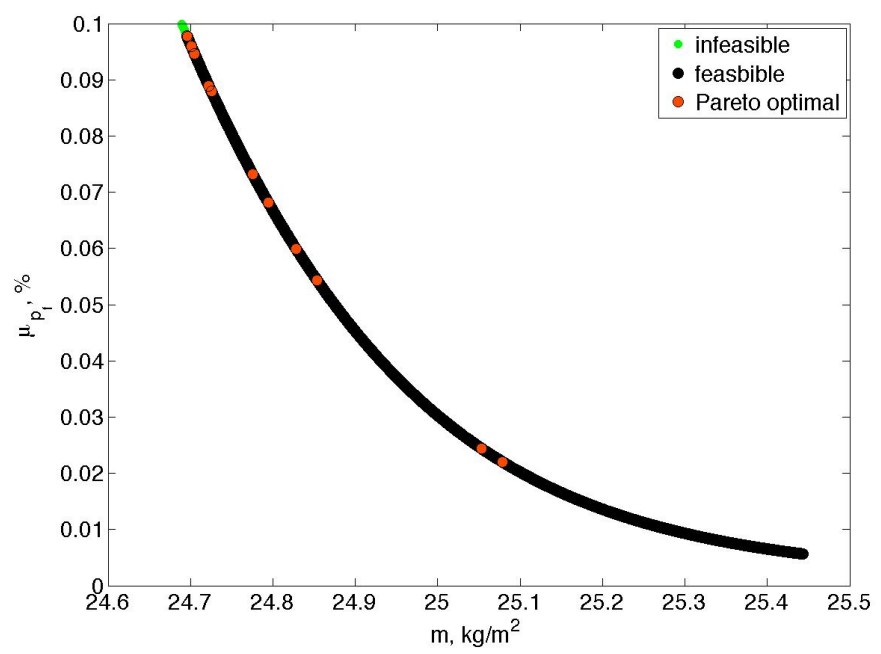

Figure 3. Pareto front for minimum mass and mean probability of failure without redesign

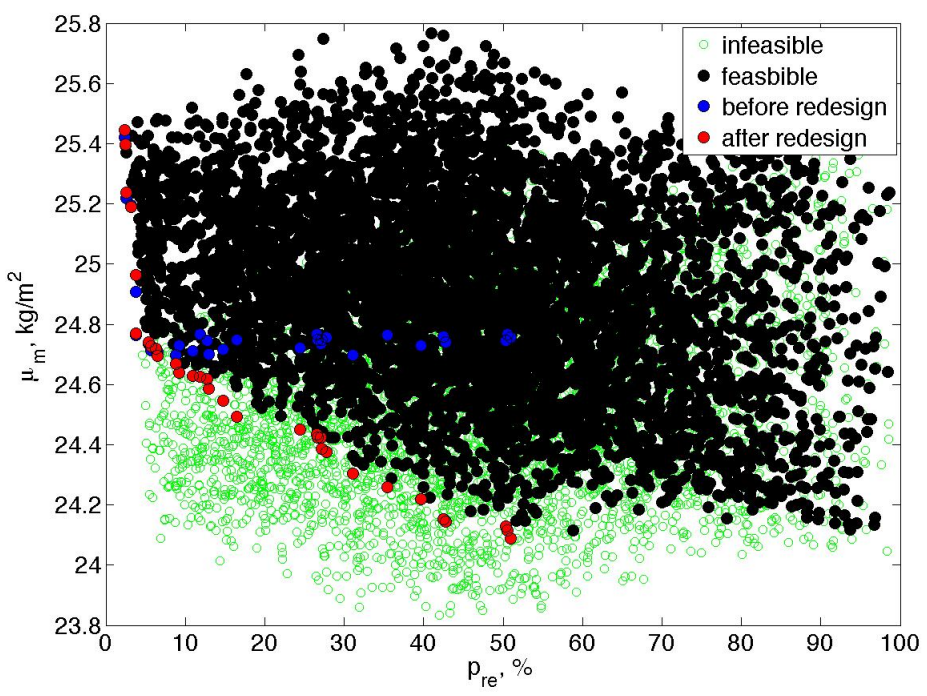

Figure 4. Pareto front for minimum probability of redesign and mean mass after redesign. Infeasible and infeasible points in the design space are shown, along with the before-redesign mass of the points on the Pareto front

The values of the safety margins for the designs on the Pareto front are displayed in Fig. 6(a). We observed that the initial safety margin $S_{1}$ had already reduced from approximately $65 \mathrm{~K}$ to $49 \mathrm{~K}$ by $p_{r e}=5 \%$. The lower bound of the acceptable safety margin with correction $S_{2}$ is at its lower bound, with a maximum value of approximately $30 \mathrm{~K}$ at probabilities of redesign less than $5 \%$. At probabilities of redesign greater than $5 \%, S_{2}$ remained between 15 to $20 \mathrm{~K}$, for which the difference from $S_{1}$ is near the upper bound of $35 \mathrm{~K}$. This resulted in the minimum probability of redesign of unconservative designs. In Fig. 6(b), which shows the percentage of the total probability of redesign that is conservative and unconservative, we observed that this was indeed the case, and that less than $5 \%$ of the total probability of redesign was attributed to probability of unconservative redesign for all points on the Pareto front.

For the upper bound on acceptable safety margin with correction $S_{3}$, we observed that the values were large (around $100 \mathrm{~K}$ ) but gradually reduced to values near $S_{1}$ at $49 \mathrm{~K}$. This led to the gradual increase in 


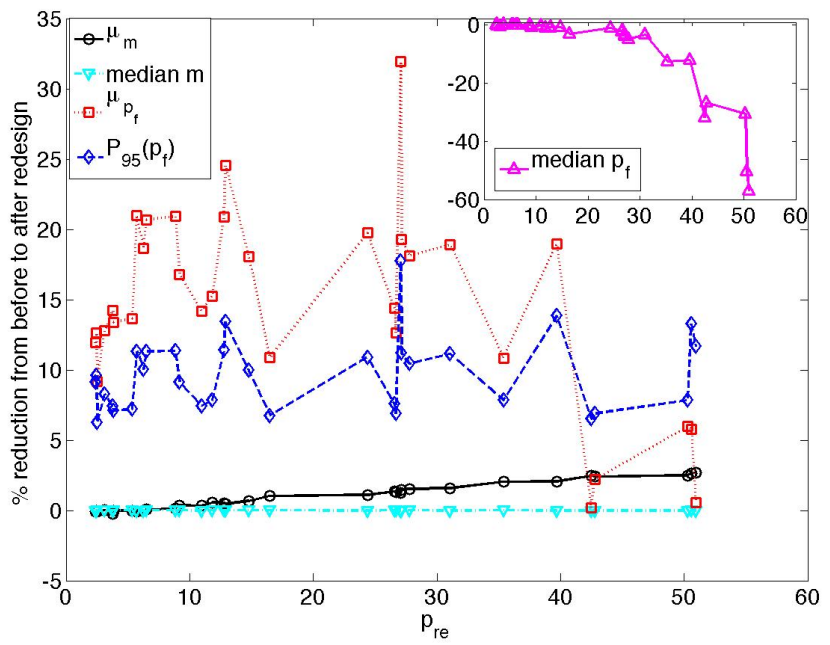

Figure 5. Percent reduction in mass and probability of failure from before to after redesign. Note that a negative reduction indicates an increase in value from before to after redesign.

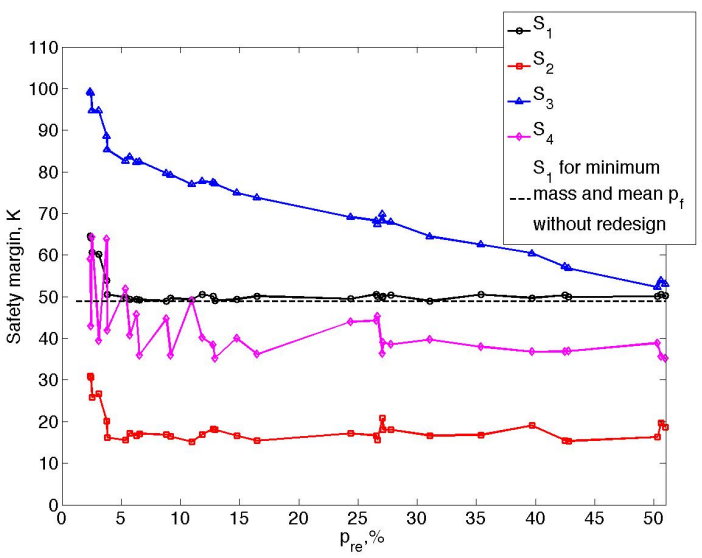

(a) Safety margin vs probability of redesign

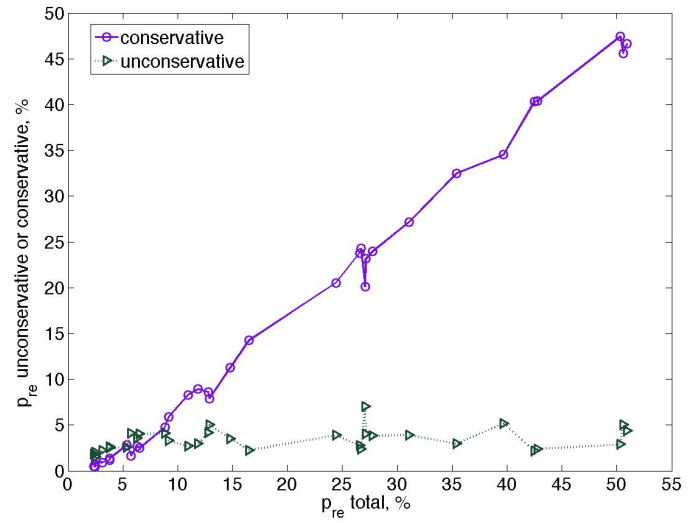

(b) Percentage of conservative and unconservative redesigns

Figure 6. For Pareto front for minimum probability of redesign and mean mass after redesign, (a) safety margins versus total probability of redesign and (b) percentage of total probability of redesign that are unconservative and conservative

probability of conservative redesign as the probability of unconservative redesign remained at low values. Thus, the probability of conservative redesign comprised the majority of the total probability of redesign for the designs on the Pareto front. At the same time, we observed that the safety margin $S_{4}$ of the redesign was set to values below $S_{1}$ and at values less than the minimum value without tests and redesign of $48.9 \mathrm{~K}$. That is, after the test, the redesign has a smaller safety margin than possible for the original design. The combined effect of redesigning conservative designs for a reduced safety margin was a reduction in the mean mass and mean probability of failure with an increase in the median probability of failure.

\section{Summary and Conclusions}

In this paper, we investigated the effect of including a future test and redesign on the optimum design of the integrated thermal protection system by tuning the variables that defined the initial design, the variables that control if redesign is performed, and and the variables that define the redesign. We examined points 
on a Pareto front for minimum probability of redesign and mean mass after redesign. At small values of probability of redesign, we initially observed that the design was slightly safer and heavier than the minimum mass design without redesign under the same constraints on safety. As the probability of redesign increased, the initial designs tended toward the minimum mass design that would be possible if redesign was not allowed. As a larger probability of redesign was accepted, the redesign criterion dictated that mostly conservative designs undergo redesign, which resulted in a reduction in the mean and 95th percentile of probability of failure and an increase in the median probability of failure. At the same time, if redesign was performed, the safety margin of the new design was less than the original, which led to a reduction in mass.

The need to keep the probability of redesign small is obvious since redesign can cause project delays and, consequently, increase costs. This study supports the idea of creating initial designs that satisfy the constraints on safety while only redesigning to reduce cost, which for the ITPS, results from reduced mass.

\section{Appendix}

\section{Integrated Thermal Protection System}

Thermal analysis of the integrated thermal protection system is done using 1-D heat transfer equations on a model of the unit cell. The heat flux incident on the top face sheet of the panel is highly dependent on the vehicle shape as well as the vehicle's trajectory. As in previous studies by Bapanapalli, ${ }^{12}$ incident heat flux on a Space Shuttle-like vehicle was used. A large portion of the heat is radiated out to the ambient by the top face sheet, and the remaining portion is conducted into the ITPS. We consider the worst-case scenario where the bottom face sheet cannot dissipate heat by assuming the bottom face sheet is perfectly insulated. Also, there is no lateral heat flow out of the unit cell, so that heat flux on the unit cell is absorbed by that unit cell only. For a more in-depth description of the model and boundary conditions, the reader is referred to the Bapanapalli reference.

The random variables used in this paper are described in Table 3.

Table 3. ITPS Random Variables

\begin{tabular}{|c|c|c|c|c|c|}
\hline Variable & Symbol & Distribution & Nominal & Bounds/ CV $(\%)$ & Correlation \\
\hline web thickness & $t_{w}$ & Uniform & - & \pm 3 & \\
\hline bottom face sheet & $t_{B}$ & Uniform & - & \pm 3 & \\
\hline foam thickness & $d_{s}$ & Uniform & - & \pm 3 & \\
\hline top face sheet thickness & $t_{T}$ & Uniform & $1.2 \mathrm{~mm}$ & \pm 3 & \\
\hline half unit cell length & $p$ & Uniform & $34.1 \mathrm{~mm}$ & \pm 3 & \\
\hline angle of corrugation & $\theta$ & Uniform & $80^{\circ}$ & \pm 3 & \\
\hline density of titanium & $\rho_{T i}$ & Normal & $4429 \frac{\mathrm{kg}}{\mathrm{m}^{3}}$ & 2.89 & correlation \\
\hline thermal conductivity of titanium & $k_{T i}$ & Normal & $7.6 \frac{W}{m / K}$ & 2.89 & coefficient $=0.95$ \\
\hline specific heat of titanium & $c_{T i}$ & Normal & $564 \frac{\mathrm{J}}{\mathrm{kg/K}}$ & 2.89 & \\
\hline density of beryllium & $\rho_{B e}$ & Normal & $1850 \frac{\mathrm{kg}}{\mathrm{m}^{3}}$ & 2.89 & correlation \\
\hline thermal conductivity of beryllium & $k_{B e}$ & Normal & $203 \frac{W}{m / K}$ & 3.66 & coefficient $=0.95$ \\
\hline specific heat of beryllium & $c_{B e}$ & Normal & $1875 \frac{\mathrm{J}}{\mathrm{kg} / \mathrm{K}}$ & 2.89 & \\
\hline density of foam & $\rho_{S}$ & Normal & $24 \frac{\mathrm{kg}}{\mathrm{m}^{3}}$ & 5.78 & correlation \\
\hline thermal conductivity of foam & $k_{S}$ & Normal & $0.105 \frac{W}{m / K}$ & 5.78 & coefficient $=0.95$ \\
\hline specific heat of foam & $c_{S}$ & Normal & $1120 \frac{\mathrm{J}}{\mathrm{kg} / \mathrm{K}}$ & 2.89 & \\
\hline initial temperature & $v_{i}$ & Uniform & 0 & 1 & \\
\hline allowable temperature & $T_{\text {allow }}$ & Lognormal & $660 \mathrm{~K}$ & 2.42 & $\left(T_{\text {allow }}^{\text {det }}=623.15 \mathrm{~K}\right)$ \\
\hline
\end{tabular}




\section{Surrogates for Mass and Probability of Failure}

In this section, it is shown that the mass before and after redesign can be found using a surrogate that is a function of safety margin and difference between the allowable temperature $T_{\text {allow }}$ and initial temperature $T_{0}$. A surrogate of of the probability of failure that is a function of the same two variables and the computational error $e_{c}$ can be made as well.

As shown in Eq.(3), the initial design satisfies

$$
T_{0}+\Delta T_{\text {calc }}(d, r)+S_{1}=T_{\text {allow }}
$$

Rearranged so that $\Delta T_{\text {calc }}(d, r)$ is on the left hand side, this becomes

$$
\Delta T_{\text {calc }}(d, r)=\left(T_{\text {allow }}-T_{0}\right)-S_{1}
$$

By Eq.(6)the redesign should satisfy

$$
T_{0}+\theta \Delta T_{\text {calc }}(d, r)+S_{4}=T_{\text {allow }}
$$

which rearranged so that $\Delta T_{\text {calc }}(d, r)$ is on the left hand side is

$$
\Delta T_{\text {calc }}(d, r)=\left(T_{\text {allow }}-T_{0}\right) / \theta-S_{4} / \theta
$$

By Eqs.(12) and (14), the two are equivalent if $\left(T_{\text {allow }}-T_{0}\right)=\left[\left(T_{\text {allow }}-T_{0}\right) / \theta\right]_{\text {afterredesign }}$ and $S_{1}=S_{4} / \theta$. Therefore, $\Delta T_{\text {calc }}$, along with its corresponding mass and probability of failure, is a function of $\left(T_{\text {allow }}-T_{0}\right)$ and $S$, where the values with and without redesign are related through $\theta$. This allows the mass to be calculated simply using surrogates with the inputs $\left(T_{\text {allow }}-T_{0}\right)$ and $S$. A surrogate to obtain the probability of failure can also be obtained by including the computational error $e_{c}$ as an input.

Note that $\Delta T_{\text {calc }}(d, r)$ does not need to be calculated because, for a given $\left(T_{\text {allow }}-T_{0}\right)$ and $S_{1}$, we can find $\Delta T_{\text {calc }}(d, r)$ by

$$
\left(T_{\text {allow }}-T_{0}\right)-S_{1}=\Delta T_{\text {calc }}(d, r)
$$

When the correction is applied, then we evaluate if redesign is necessary by

$$
\begin{aligned}
\text { Redesign if: } & \left(T_{\text {allow }}-T_{0}\right)-\theta\left[\left(T_{\text {allow }}-T_{0}\right)-S_{1}\right] \leq S_{2} \\
& \text { or }\left(T_{\text {allow }}-T_{0}\right)-\theta\left[\left(T_{\text {allow }}-T_{0}\right)-S_{1}\right] \geq S_{3}
\end{aligned}
$$

which simplifies to

$$
\begin{aligned}
\text { Redesign if: } & \left(T_{\text {allow }}-T_{0}\right)(1-\theta)+\theta S_{1} \leq S_{2} \\
& \text { or }\left(T_{\text {allow }}-T_{0}\right)(1-\theta)+\theta S_{1} \geq S_{3}
\end{aligned}
$$

\section{Detailed Results}

Pareto front for minimum mass and probability of failure without redesign

The Pareto front for minimum probability of failure and mass without redesign is described in detail in Table 4.

Pareto front for minimum probability of redesign and mean mass after redesign

The Pareto front for minimum probability of redesign and mean mass after redesign is described in detail in Table 5 .

\section{Acknowledgments}

The material is based upon work supported by NASA under award No.NNX08AB40A. Any opinions, findings, and conclusions or recommendations expressed in this material are those of the author(s) and do not necessarily reflect the views of the National Aeronautics and Space Administration. 
Table 4. Pareto front for minimum mass and mean probability of failure without redesign

\begin{tabular}{ccccc}
\hline$S_{1}(\mathrm{~K})$ & $\mathrm{m}\left(\mathrm{kg} / \mathrm{m}^{2}\right)$ & $\mu_{p_{f}}(\%)$ & $P_{95}\left(p_{f}\right)(\%)$ & Median $p_{f}(\%)$ \\
\hline 57.7 & 25.08 & 0.022 & 0.117 & $2.05 \mathrm{E}-05$ \\
57.1 & 25.05 & 0.024 & 0.129 & $2.58 \mathrm{E}-05$ \\
52.6 & 24.85 & 0.054 & 0.280 & $1.71 \mathrm{E}-04$ \\
52.0 & 24.83 & 0.060 & 0.308 & $2.16 \mathrm{E}-04$ \\
51.2 & 24.79 & 0.068 & 0.350 & $2.98 \mathrm{E}-04$ \\
50.8 & 24.78 & 0.073 & 0.376 & $3.54 \mathrm{E}-04$ \\
49.6 & 24.73 & 0.088 & 0.450 & $5.55 \mathrm{E}-04$ \\
49.5 & 24.72 & 0.089 & 0.455 & $5.70 \mathrm{E}-04$ \\
49.2 & 24.71 & 0.095 & 0.484 & $6.64 \mathrm{E}-04$ \\
49.1 & 24.70 & 0.096 & 0.490 & $6.86 \mathrm{E}-04$ \\
49.0 & 24.70 & 0.098 & 0.499 & $7.16 \mathrm{E}-04$ \\
48.9 & 24.70 & 0.098 & 0.500 & $7.19 \mathrm{E}-04$ \\
48.9 & 24.70 & 0.098 & 0.500 & $7.19 \mathrm{E}-04$ \\
\hline
\end{tabular}

\section{References}

\footnotetext{
${ }^{1}$ Fujimoto, Y., C., K. S., Hamada, K., and Huang, F., "Inspection Planning Using Genetic Algorithm for Fatigue Deteriorating Structures," International Offshore and Polar Engineering Conference, Vol.4, International Society of Offshore and Polar Engineers, Golden, CO, 1998, pp. 99-109.

${ }^{2}$ Toyoda-Makino, M., "Cost-based Optimal History Dependent Strategy for Random Fatigue Cracks Growth," Probabilistic Engineering Mechanics, Vol. 14, No. 4, October 1999, pp. 339-347.

${ }^{3}$ Garbatov, Y. and Soares, C. G., "Cost and Reliability Based Strategies for Fatigue Maintenance Planning of Floating Structures," Reliability Engineering and Systems Safety, Vol. 73, No. 3, 2001, pp. 293-301.

${ }^{4}$ Kale, A., Haftka, R. T., and Sankar, B. V., "Efficient Reliability-Based Design and Inspection of Panels Against Fatigue," Journal of Aircraft, Vol. 45, No. 1, 2008, pp. 86-96.

${ }^{5}$ Acar, E., Haftka, R. T., and Kim, N. H., "Effects of Structural Tests on Aircraft Safety," AIAA Journal, Vol. 48, No. 10, 2010.

${ }^{6}$ Sankararaman, S., McLemore, K., Liang, C., Bradford, S. C., and Peterson, L., "Test resource allocation for uncertainty quantification of multi-level and coupled systems," 52nd AIAA/ASME/ASCE/AHS/ASC Structures, Structural Dynamics, and Materials Conference, Denver, CO, 2011.

${ }^{7}$ Villanueva, D., Haftka, R. T., and Sankar, B. V., "Including the Effect of a Future Test and Redesign in Reliability Calculations," AIAA Journal, Vol. 49, No. 12, 2011, pp. 2760-2769.

${ }^{8}$ Matsumura, T., Haftka, R. T., and Sankar, B. V., "Reliability Estimation Including Redesign Following Future Test for an Integrated Thermal Protection System," 9th World Congress on Structural and Multidisciplinary Optimization, Shizuoka, Japan, 2011.

${ }^{9}$ Oberkampf, W. L., Deland, S. M., Rutherford, B. M., Diegert, K. V., and Alvin, K. F., "Error and Uncertainty in Modeling and Simulation," Reliability Engineering and System Safety, Vol. 75, 2002, pp. 333-357.

${ }^{10}$ Acar, E., Haftka, R. T., Kim, N. H., and Buchi, D., "Effects of structural tests on aircraft safety," 50th AIAA/ASME/ASCE/AHS/ASC Structures, Structural Dynamics, and Materials Conference, Palm Springs, CA, May 2009.

${ }^{11}$ Smarslok, B. P., Haftka, R. T., Carraro, L., and Ginsbourger, D., "Improving accuracy of failure probability estimates with separable Monte Carlo," International Journal of Reliability and Safety, Vol. 4, 2010, pp. 393-414.

${ }^{12}$ Bapanapalli, S. K., Design of an Integrated Thermal Protection System for Future Space Vehicles, Ph.D. thesis, University of Florida, 2007.
} 


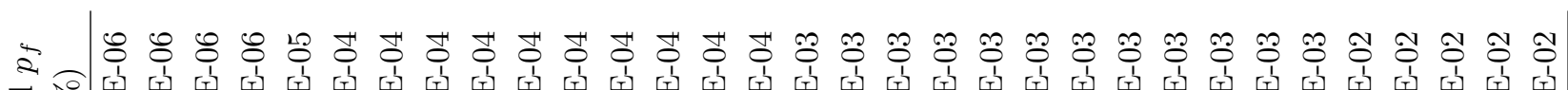

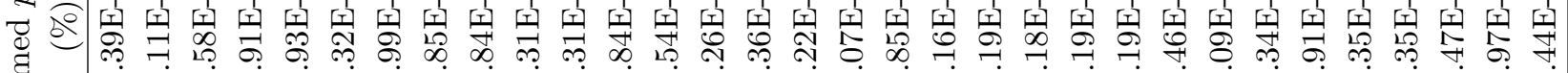
కి

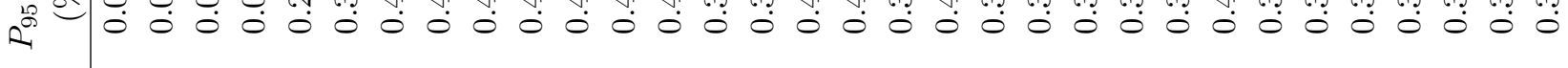
总

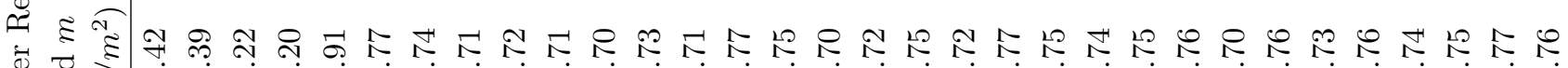
女

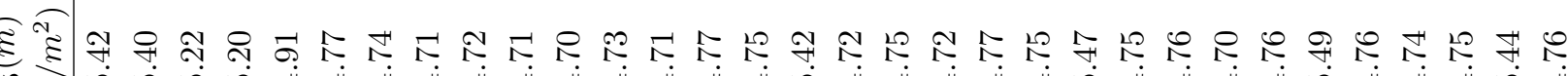
20 กิ

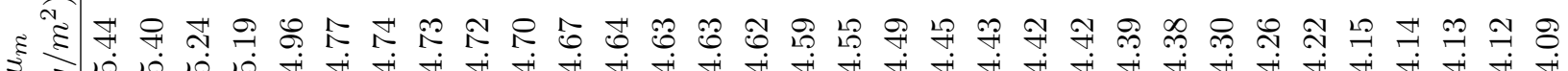
ई

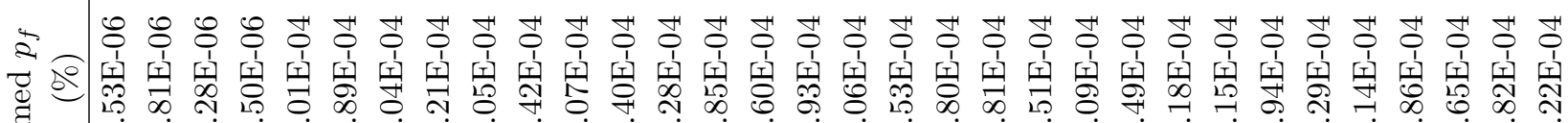

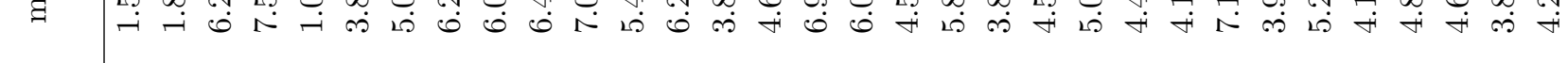
कี

苞

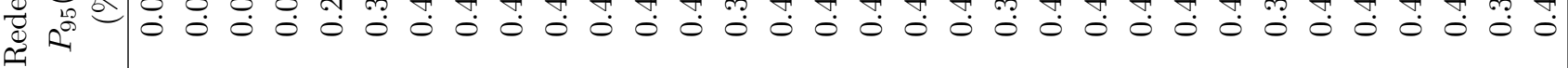
至

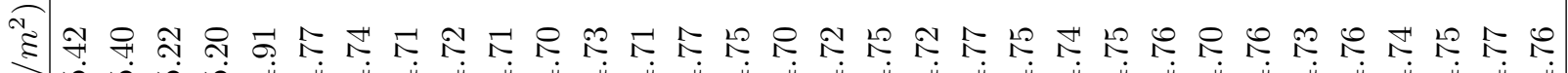
产| 㻤

节

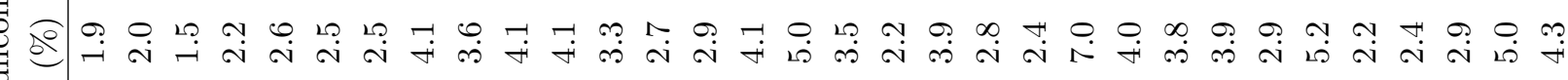
剀

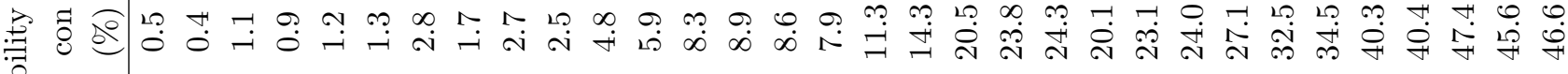
$\frac{\pi}{0}$

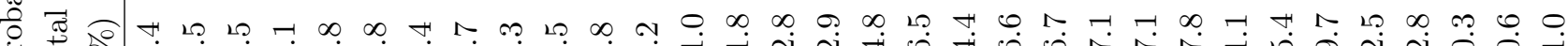

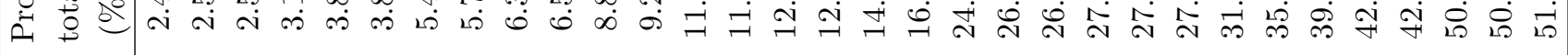

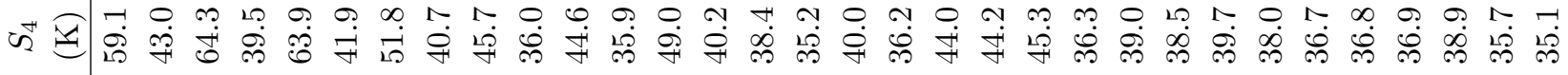
$\frac{2}{9}$

๘ 苛

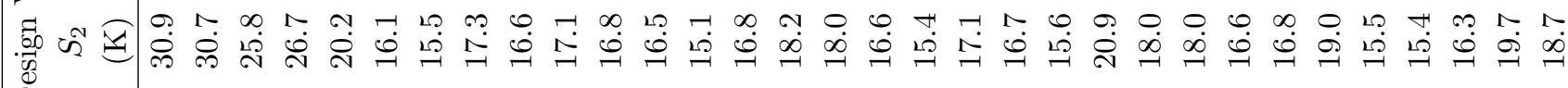
คั

क 